\title{
RANS Simulation and Experiments on the Stall Behaviour of a Tailplane Airfoil
}

\author{
R. WOKOECK ${ }^{1}$, A. GROTE ${ }^{1}$, N. KRIMMELBEIN ${ }^{1}$, J. ORTMANNS ${ }^{1}$, R. RADESPIEL ${ }^{1}$ \\ and A. KRUMBEIN ${ }^{2}$ \\ ${ }^{1}$ Institute of Fluid Mechanics, Braunschweig Technical University, \\ Bienroder Weg 3, 38106 Braunschweig, Germany \\ e-mail: r.wokoeck@tu-bs.de \\ ${ }^{2}$ Institute of Aerodynamics and Flow Technology, German Aerospace Center DLR, \\ Lilienthalplatz 7, 38108 Braunschweig, Germany \\ e-mail: andreas.krumbein@dlr.de
}

\begin{abstract}
Summary
Measurements and simulations are presented of the flow past a tailplane research airfoil which is designed to show a mixed leading-edge trailing-edge stall behaviour. The numerical simulations were carried out with two flow solvers that introduce transition prediction based on linear stability theory to RANS simulations for cases involving laminar separation bubbles. One of the methods computes transition locations across laminar separation bubbles whereas the other assumes transition onset where laminar separations occur. For validation of the numerical methods an extensive measurement campaign has been carried out. It is shown, that the methodology mentioned first can simulate the size of laminar separation bubbles for angles of attack up to where the separation bubble and the turbulent separation at the trailing edge are well behaved and steady in the mean. With trailing edge separation involved, the success of the new numerical procedure relies on the diligent choice of a turbulence model. Finally, for flows with increased unsteady behaviour of both, separation bubble and turbulent separation, which were observed at higher angles of attack in the experiment between maximum lift and leading-edge stall, steady state prediction methods for transition can no longer be applied and time-accurate methods have to be developed in a further step.
\end{abstract}

\section{Introduction}

For calculating the maximum lift of airfoils with a laminar separation bubble close to the leading edge, the precise prediction of transition location along the bubble is important. According to the classification [3] of the stall-types with leading-edge separation bubbles involved, the separation bubble at large angles of attack can either burst causing the so-called leading-edge stall or - for higher Reynolds numbers - will lead to a mixture of leading and trailing edge stall by interacting with the turbulent separation that is moving upstream from the trailing edge before the burst. For the latter case, various associated phenomena have been observed: The trailing-edge separation can be of strong unsteady 
character $[4,5]$. The separated area near the trailing-edge viewed in wind-tunnel experiments usually appears in a spanwise irregular shape [6] and bears inside three-dimensional cells of circulating flow $[16,10]$.

Widely used numerical procedures consist of utilising a boundary-layer code for calculating the viscous, surface near flowfield in interaction with the inviscid outer flowfield $[8,9]$. Determination of the transition position is handled either by databases of solutions of the Orr-Sommerfeld differential equation or by envelope-methods derived from such databases. Also semi-empirical approaches are used to determine the bubble size [17] and burst [2]. The prediction of transition on a laminar separation bubble is new in the context of flow solvers based on the Reynolds-averaged Navier-Stokes equations (RANS). Addressing this subject, the Institute of Fluid Mechanics (ISM) of Braunschweig Technical University has developed a methodology that couples a RANS flow solver to a stability method for the Tollmien-Schlichting instabilities, which occur in the 2D shear layer. For obtaining a solid and comprehensive data base for validation purposes, a dedicated research airfoil was designed, built and tested in a subsonic wind tunnel.

\section{Design of the research airfoil}

The aerodynamic design of the new research airfoil, named HGR-01, was defined to realize the mixed stall type. This was challenging due to the lack of adequate tools for predicting these stall types (as the acquirement of the latter is the matter of subject of the project). The chosen modus operandi described in [11] was to use the panel-method code XFOIL [9] for step-by-step reproduction of the near-nose pressure distribution of members of the well documented LWK airfoil family [1], which have shown the desired stall behaviour with laminar separation bubbles involved.

\section{Experimental set up}

The model of the research airfoil was manufactured of carbon fibre reinforced epoxy with a flap size of $30 \%$ chord for influencing the pressure distribution at the nose and so affecting the stall behaviour. It features 55 pressure tabs distributed on the surface with higher density at the nose and around the upper leading-edge area for resolving transition and separation of the flow. The experiments took place in the subsonic wind tunnel MUB of ISM with a test section of $1.3 \mathrm{~m}$ squared and a characteristic turbulence level of about $0.2 \%$. The experiments provided data for three different Reynolds numbers: $0.35,0.7$ and 1.4 million. The angle of attack was varied from negative lift to beyond stall. With the help of $2 \mathrm{~cm}$ long silk tufts attached in a $2 \mathrm{~cm}$ spacing a quick overview of the flow behaviour at different angles of attack was achieved. Also the tufts were chosen for measuring the turbulent separation. Then the more inert technique of oil-flow visualization that delivers a far better spatial resolution of 
the surface friction was applied. With oil flow the transition line for low angles of attack as well as the occurrence, position and size of the laminar separation bubble at higher angles were determined. Oil was also used to visualize the structures within the separated area and to detect the unwanted interaction between the boundary layers of the tunnel side walls and the flow past the model at very high angles of attack. The surface pressure distribution in the centre region of the model was delivered by the pressure tabs. Lift and drag were to be derived according to Jones' approach with the pressure distribution in the wake to be measured by pressure rakes. Due to strong unsteadiness in the wake at high angles of attack the present work utilizes only the pressure-induced normal force coefficient, $\mathrm{C}_{\mathrm{n}, \mathrm{p}}$ for comparison to numerical results instead of the more commonly used lift and drag coefficients. It should be mentioned, that the curve of the pressure-induced normal force is very similar to the lift curve. $\mathrm{C}_{n, p}$ is derived by integrating the measured pressure distribution shown exemplarily in fig. 5 around the surface. With the existing pressure tabs resolution obviously being too coarse for resolving the lower side pressure correctly, a correction (adding the tinted areas) to the measured normal force has been applied. This correction was deduced by projecting the measured pressure values on the numerical pressure distribution for several angles of attack so that the true differences between the measured and the calculated pressure forces are preserved. Finally, the non-intrusive method PIV was applied for investigation of characteristics in the area of turbulent trailing edge separation. For each flowfield measurement 1000 pictures illuminated by two Quantech brilliant Nd:YAG lasers with $150 \mathrm{~mJ}$ oul output per pulse at $532 \mathrm{~nm}$ were taken by a peltier cooled LaVision Flowmaster camera with a resolution of 1280x1024 pixels and an effective $360 \mathrm{~mm}$ focal length. This recording system was swivel mounted following the angle of attack to assure constant display windows. For processing the velocity fields, the Davis 6.2 software of LaVision was applied with an iterative multigrid scheme of second order and $0 \%$ overlap.

\section{Numerical methods}

\subsection{Methodology of transition prediction of ISM}

The transition prediction methodology developed by ISM is carried out via a coupled program system shown in fig. 1. This system consists of the RANS solver TAU [7] originated by German Aerospace Center (DLR) that is coupled with a stability method based on the linear stability theory via the transition prediction module. The transition prediction module interrupts the RANS solver TAU after a certain number of iterations (which can be decided, for example, upon a residual) performed with a transition position defined by the user as initial guess. The module extracts all information of the actual boundary-layer flow field necessary for the stability method, hands it over and starts this method. The output returned by the linear stability method consists of the $\mathrm{N}$-factors for the amplified frequencies and is analysed by the transition prediction module that generates an envelope above all $\mathrm{N}$-factors and compares this to a critical value. 
This value is determined by free-stream conditions and can be found for example via Mack's formula [14]. The new transition position is assumed to be located where this value is exceeded and is then returned with under-relaxation into the flow field of the solver TAU for restart, closing one work loop. The number of loops can be fixed by the user or decided upon the convergence of the transition positions between work loops. As stability method the fast and proven stability solver COAST3 by G. Schrauf [18] is chosen. The transition length itself is then determined by the growth of the turbulent Reynolds stresses, as given by the turbulence model used in the RANS solver. Previous investigations have shown that, for this setup, a resolution of the laminar boundary-layer normal to the surface with at least 25 grid points is necessary. To fulfil this condition, a set of three grids with different overall resolution for grid refinement study purposes was generated for the computations. The grids (see tab. 1) were specially refined in areas of interest, and the medium grid resolution (fig. 11) was found to be sufficient for resolving the laminar separation bubble and the turbulent separation.

\subsection{Methodology of transition prediction of DLR}

The methodology developed and applied for the computations of DLR is described in detail in another article of this book [12] and has been published in [13]. Thus, only the differences compared to the methodology of ISM shall be highlighted here: Instead of feeding the flow field of the RANS Solver (here FLOWer) directly into the stability method, the necessary boundary-layer data are obtained by a boundary-layer method, that is fed with the pressure field generated by the RANS solver. This procedure allows for much coarser grid resolution (see fig. 9 and table 1) compared to ISM methodology. As stability method, a database by Degenhart and Stock [19] for the Tollmien-Schlichting instabilities is consulted. Depending on a boundary-layer method, this methodology can only analyze the region up to the laminar separation for stability. So, for laminar separation bubbles, contrary to the ISM methodology, here the point of laminar separation is utilized as transition location and an intermittency function and algebraic transition length for modelling the transitional flow regions is applied.

\section{Results and Discussion}

As the amount of data acquired in experiment and numerical simulation can not extensively be covered here, along with the general description of the overall performance of the research airfoil, the Reynolds number 0,7 million case is chosen as an example of the observed flow phenomena. Detailed insight and comparisons to numerical results are then given for $12^{\circ}$ angle of attack. Flap deflection and its associated flow phenomena are beyond the scope of this article and therefore not covered here. For all three Reynolds numbers measured at relatively low, positive angles of attack (alpha), the flow on the upper surface 
(suction side) is fully attached with the transition marching upstream with increasing angle. The laminar separation bubble occurs in the nose area at medium angles long before the turbulent separation at the trailing edge starts. Figure 7 gives an example of the flow past the upper surface of the model visualized with oil paint. The extent of the separation bubble is clearly visible as a straight line over the model span as there is paint captured within the bubble through the experiment duration. In general, the separation bubble shrinks and moves towards the nose as the angle of attack is increased (see fig. 3 ) and it is larger for lower Reynolds numbers (not shown here). With further increased angle, the turbulent separation starts from the trailing edge and moves upstream (see fig. 4). At this point the pressure-induced normal force in fig. 2 deviates from the linear distribution. The growth of the separated area is what limits the maximum normal force (and thereby the maximum lift) in this mixed stall behaviour, that appears at all three Reynolds numbers. For higher Reynolds numbers the maximum normal force coefficient is larger (not shown here). After the location of the turbulent separation has moved to upstream to about $75 \%$ of the chord the flow changes for higher angles to a rather unsteady character revealed by the quick tufts and PIV. The turbulent separation then moves back and forth in between $25 \%$ and $75 \%$ of the chord. The full airfoil stall is then obtained for even higher alphas by the burst of the separation bubble, which takes place earlier (lower angles) for lower Reynolds numbers (not shown here). Here, a significant hysteresis is measured in the normal force. That is, leadingedge stall occurs at larger angles for increasing alpha whereas it occurs at smaller angle for decreasing alpha. Several owl-eyes-called patterns fill the area of turbulent separation depicted in fig. 8. These structures remain uniform and even in number as long as the separated area is small but they change into an uneven low number in the unsteady phase mentioned above and strongly interact with the tunnel side-wall boundary layers. This interaction has been observed in many wind tunnels. It impairs the two-dimensional character of the flow and thereby affects the measurements - a problem, that will be investigated and hopefully reduced by tangentially blowing air into the junction of airfoil and side wall during future experimental work.

The normal forces yielded by the two numerical methods for the 0.7 million Reynolds number case show the same slope up to angles of attack of $10^{\circ}$ (fig. 2). For the computations run with ISM methodology and $\alpha \leq 10^{\circ}$ position and size of the laminar separation bubbles match the experimental data resolved by oil and pressure measurements very well (fig. 3). Pressure distributions of ISM computations for $\alpha \leq 10^{\circ}$ are also in good agreement with the experiment for the whole surface (not shown here). The ISM computation at $\alpha=12^{\circ}$ had to be performed in the time-accurate mode with rather small time steps $\Delta \mathrm{t}\left(\right.$ chord $\left./ \mathrm{U}_{\infty}\right)=0.25 \%$ in order to resolve the fluid motion in space and time. However, this way an almost steady pressure distribution was obtained as shown in figure 6. Note that the fine mesh with 82559 nodes gave almost identical results as the medium mesh with 46462 nodes. The computations at $12^{\circ}$ with DLR methodology did not yield a separation bubble (fig. 6 and 10). This may be caused by the much coarser grid and by the fact, that transition onset is forced at 
laminar separations. The experimentally viewed unsteady phase around $12^{\circ}$ and the onset of leading-edge stall is not rendered correctly by any of the numerical setups yet (fig. 2). For higher angles both DLR and ISM computations could only be carried out to convergence using the time accurate mode of the RANS solvers. These DLR computations at high angles show irregular oscillations of the normal force [12]. In the computations with the ISM method for $\alpha>10^{\circ}$ the chosen Spalart-Allmaras (SA) turbulence model underpredicts the turbulent separation (figs. 13, 14), which leads to a higher suction peak at the nose, and larger overall suction on the upper side (fig. 6). The DLR computation for $12^{\circ}$ does not show any turbulent separation either (not shown here). This reveals the necessity to identify a turbulence model that resembles the experimentally observed flow correctly. First improvements could be found with the SALSA turbulence model [15] (fig. 4) but more potential is expected from currently tested, more advanced second-moment closure Reynolds stress models (RSM), which in addition deliver the turbulent shear stresses directly comparable to the particle image measurements. It may appear, that the unsteady behaviour of the laminar separation bubble and the turbulent separation and finally the burst itself can only be captured if the temporal history of the amplification rates is taken into account by a time-accurate formulation of the $\mathrm{e}^{\mathrm{N}}$ method. This is an extension of the numerical method that will be attempted in future work.

\section{Acknowledgment}

The numerical and experimental investigations were partly founded by the NRC-Helmholtz Collaborative Research Program and by Airbus Deutschland $\mathrm{GmbH}$. This support is thankfully acknowledged by the authors.

\section{References}

[1] Althaus, D.: "Niedriggeschwindigkeitsprofile", Vieweg, Braunschweig, 1996.

[2] Baragona, M., Boermanns, L. M. M., van Tooren M. J. L., Bijl H., Beukers A.: "Bubble Bursting and Stall Hysteresis on Single-Slotted Flap High Lift Configuration", AIAA Journal, Vol. 41 (2003), No. 7, pp. 1230-1237.

[3] van den Berg, B.: "Reynolds number and Mach number effects on the maximum lift and stalling characteristics of wings at low speeds", NLR TR 69025 U, Amsterdam, Netherlands 1969.

[4] Bragg, M. B., Heinrich, D. C., Balow, F.A.: "Flow Oscillation over an Airfoil Near Stall", AIAA Journal, Vol. 34 (1994), No. 1, pp. 199-201.

[5] Broeren, A. P., Bragg, M. B.: "Flowfield Measurements over an Airfoil During Natural Low-Frequency Oscillations near Stall", AIAA Journal, Vol. 37 (1999), No. 1, pp. 130-132.

[6] Broeren, A. P., Bragg, M. B.: "Spanwise Variation in the Unsteady Stalling Flowfields of Two-Dimensional Airfoil Models", AIAA Journal, Vol. 39 (2001), No. 9, pp. 1641-1651.

[7] DLR, Institute of Aerodynamics and Flow Technology: "TAU-Code User Guide Revision: 1.24, Release 2004.1.0", April 5, 2004. 
[8] Drela, M.: "Two-Dimensional Transonic Aerodynamic Design and Analysis Using the Euler Equations, Ph.D. Dissertation, Dept. of Aeronautics and Astronautics, Massachusetts Inst. Of Technology, Cambridge, MA, 1985.

[9] Drela, M.: "XFOIL: An analysis and design system for low Reynolds number airfoils" in: Mueller, T.J. (Ed.): "Low Reynolds number aerodynamics", Springer (Lecture notes in engineering), 1989, pp. 1-12.

[10] Gleyzes, C., Capbern P.: "Experimental study of two AIRBUS/ONERA airfoils in near stall conditions. Part I: Boundary layers", Aerospace Sience and Technology 7 (2003), pp. 439-449.

[11] Grote, A., Ortmanns J., Radespiel, R.: "Entwurf eines Höhenleitwerkprofils mit Leading-Edge Stall und Konstruktion eines Windkanalmodells mit Ruder für die Messung der Profileigenschaften", Institutsbericht 2003/2, Institut für Strömungsmechanik, TU Braunschweig, 2003.

[12] Krumbein, A.: "Navier-Stokes Airfoil Computations with Automatic Transition Prediction using the DLR TAU code - A Sensitvity Study", $14^{\text {th }}$ Symposium of STAB, Bremen 2004.

[13] Krumbein, A.: "Automatic Transition Prediction and Application to High-Lift Multi-Element Configurations", AIAA-2004-2543, 34 ${ }^{\text {th }}$ AIAA Fluid Dynamics Conference, Portland, Oregon, USA, June/July 2004 (accepted for publication in Journal of Aircraft).

[14] Mack, L. M.: "Transition and Laminar Instability", Jet Propulsion Laboratory Publication 77-15, Pasadena, CA, 1977.

[15] Rung, T., Bunge, U. ,Schatz, M., Thiele, F.: "Restatement of the Spalart-Allmaras Eddy-Viscosity Model in Strain-Adaptive Formulation", AIAA Journal, Vol. 41 (2003), No. 7, pp. 1396-1399.

[16] Schewe, G.: "Reynolds-number effects in flow around more-or-less bluff bodies", Journal of Wind Engineering and Industrial Aerodynamics 89 (2001), pp. 12671289.

[17] Schmidt, G. S., Mueller, T. J.: "Analysis of Low Reynolds Number Separation Bubbles Using Semiempirical Methods", AIAA Journal, Vol. 27 (1989), No. 8, pp. 993-1001.

[18] Schrauf, G.: "COAST3 - A compressible stability code. User's guide and tutorial", Daimler-Benz Aerospace Airbus GmbH, Bremen, Technical Report EF 040/98, 1998.

[19] Stock, H. W., Degenhart, E.: "A simplified $\mathrm{e}^{\mathrm{n}}$ method for transition prediction in two-dimensional, incompressible boundary layers", Z. Flugwissenschaft Weltraumforschung 13, 1989. 
Table 1 Grids

\begin{tabular}{|c|c|c|c|c|c|}
\hline \multirow{2}{*}{ grid } & \multicolumn{4}{|c|}{ structured area } & \multirow{2}{*}{$\begin{array}{c}\text { hybrid area } \\
\text { nodes }\end{array}$} \\
\hline & $\begin{array}{c}\text { resolution } \\
\text { tangential } x \text { normal }\end{array}$ & $1^{\text {st }}$ spacing & height & nodes & \\
\hline ISM-coarse & $288 \times 48$ & $2.1 \times 10^{-5}$ & 0.012 & 11759 & 17832 \\
\hline ISM-medium & $576 \times 96$ & $1.05 \times 10^{-5}$ & 0.012 & 46462 & 55535 \\
\hline ISM-fine & $768 \times 128$ & $7.875 \times 10^{-6}$ & 0.012 & 82559 & 93195 \\
\hline DLR & $256 \times 73$ & $2.5 \times 10^{-5}$ & up to farfield & 32466 & structured \\
\hline
\end{tabular}

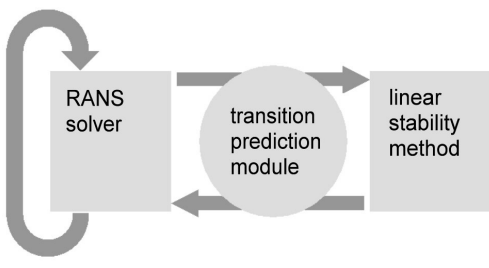

Figure 1 Coupled program system

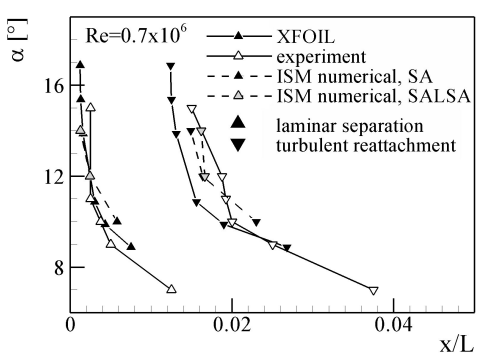

Figure 3 Laminar separation bubble

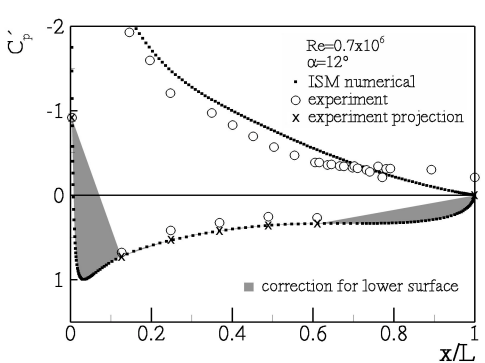

Figure 5 Pressure distribution correction of $\mathrm{C}_{\mathrm{n}, \mathrm{p}}$

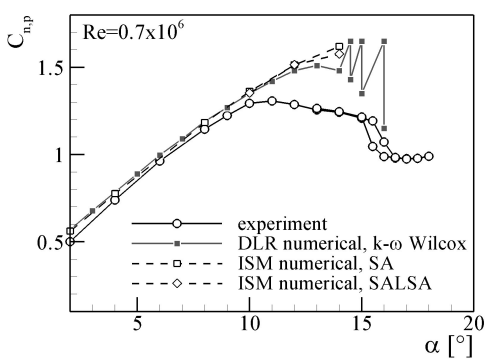

Figure $2 C_{n, p}$

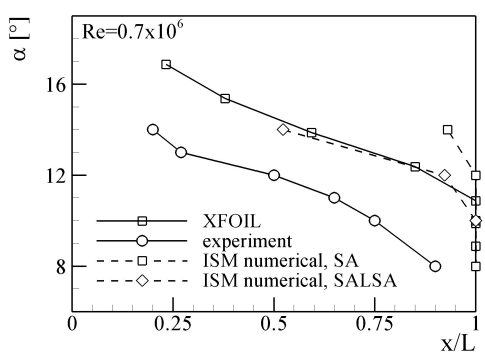

Figure 4 Turbulent separation

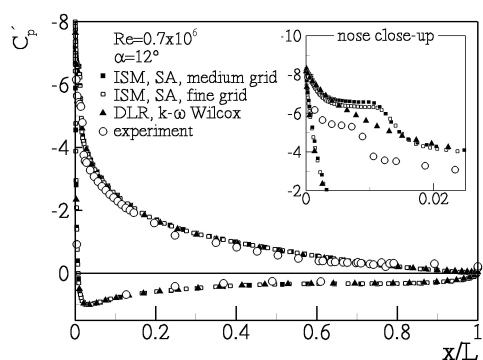

Figure 6 Pressure distribution 


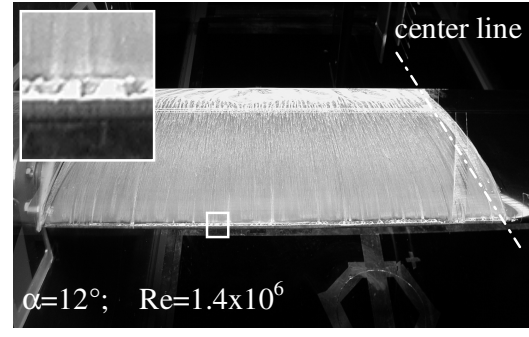

Figure 7 Oil flow visualization front view

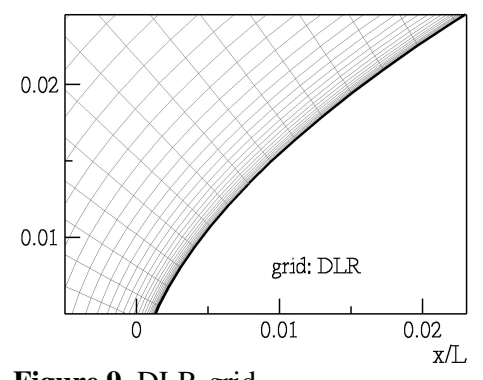

Figure 9 DLR-grid nose region

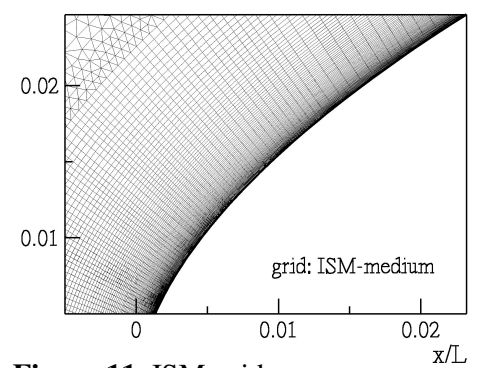

Figure 11 ISM-grid nose region

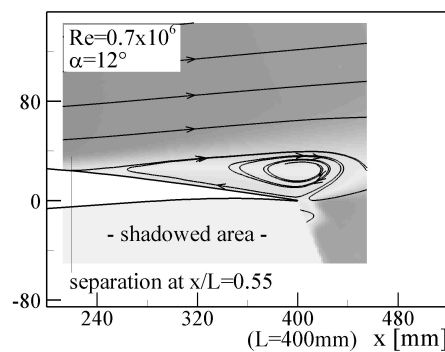

Figure 13 PIV-measurement trailing edge region

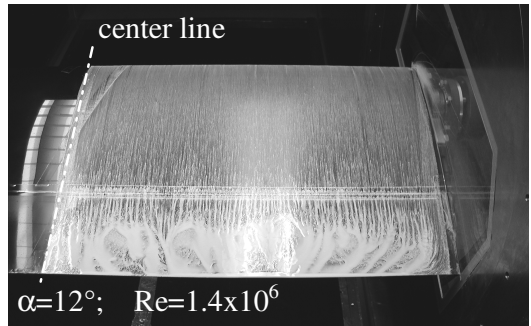

Figure 8 Oil flow visualization rear view

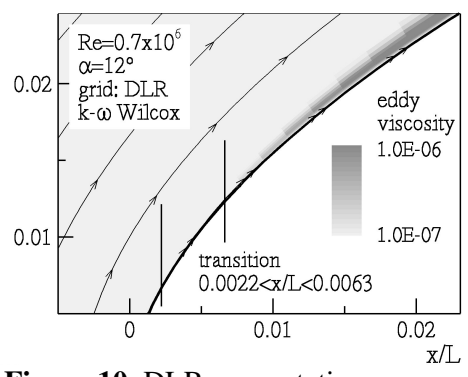

Figure 10 DLR-computation nose region

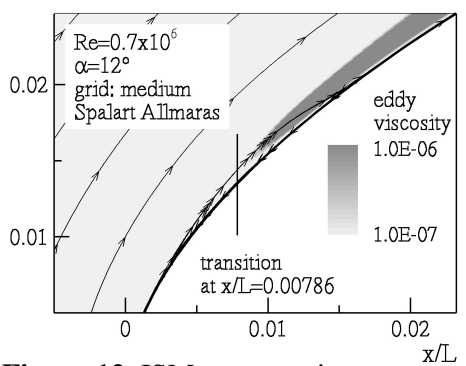

Figure 12 ISM-computation nose region

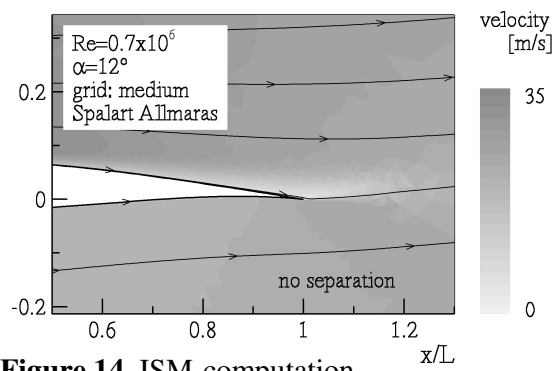

Figure 14 ISM-computation trailing edge region 